KYUNGPOOK Math. J. 53(2013), 467-478

http://dx.doi.org/10.5666/KMJ.2013.53.3.467

\title{
New Subclasses of Harmonic Starlike and Convex Functions
}

\author{
SAurabH PorwaL* \\ Department of Mathematics, U. I. E. T. Campus, C. S. J. M. University, Kanpur- \\ 208024, (U. P.), India \\ e-mail : saurabhjcb@rediffmail.com \\ Kaushal Kishore Dixit \\ Department of Engineering Mathematics, Gwalior Institute of Information Tech- \\ nology, Gwalior-474015, (M. P.), India \\ e-mail : kk.dixit@rediffmail.com
}

ABstract. The purpose of the present paper is to establish some interesting results involving coefficient conditions, extreme points, distortion bounds and covering theorems for the classes $V_{H}(\beta)$ and $U_{H}(\beta)$. Further, various inclusion relations are also obtained for these classes. We also discuss a class preserving integral operator and show that these classes are closed under convolution and convex combinations.

\section{Introduction}

A continuous complex-valued function $f=u+i v$ is said to be harmonic in a simply connected domain $D$ if both $u$ and $v$ are real harmonic in $D$. In any simply connected domain we can write $f=h+\bar{g}$, where $h$ and $g$ are analytic in $D$. We call $h$ the analytic part and $g$ the co-analytic part of $f$. A necessary and sufficient condition for $f$ to be locally univalent and sense-preserving in $D$ is that $\left|h^{\prime}(z)\right|>\left|g^{\prime}(z)\right|, z \in D$. See Clunie and Sheil-Small [3], for more basic results on harmonic functions one may refer to the following standard introductory text book by Duren [7], see also Ahuja [1] and Ponnusamy and Rasila ([9], [10]).

Let $S_{H}$ denote the class of functions $f=h+\bar{g}$ that are harmonic univalent and sense-preserving in the open unit disk $U=\{z:|z|<1\}$ for which $f(0)=$ $f_{z}(0)-1=0$. Then for $f=h+\bar{g} \in S_{H}$ we may express the analytic functions $h$ and $g$ as

$$
h(z)=z+\sum_{k=2}^{\infty} a_{k} z^{k}, g(z)=\sum_{k=1}^{\infty} b_{k} z^{k}, \quad\left|b_{1}\right|<1
$$

* Corresponding Author.

Received October 4, 2011; accepted November 7, 2012.

2010 Mathematics Subject Classification: 30C45, 30C50.

Key words and phrases: Harmonic, analytic, univalent, starlike and convex functions. 
Note that $S_{H}$ reduces to class $S$ of normalized analytic univalent functions if the co-analytic part of its member is zero. In fact, Clunie and Sheil-Small [3] investigated the class $S_{H}$.

A function $f \in S_{H}$ is said to be harmonic starlike of order $\alpha(0 \leq \alpha<1)$ in $U$, if and only if

$$
\operatorname{Re}\left\{\frac{z h^{\prime}(z)-\overline{z g^{\prime}(z)}}{h(z)+\overline{g(z)}}\right\} \geq \alpha, \quad z \in U,
$$

and is said to be harmonic convex of order $\alpha(0 \leq \alpha<1)$ in $U$, if and only if

$$
\operatorname{Re}\left\{1+\frac{z^{2} h^{\prime \prime}(z)+\overline{2 z g^{\prime}(z)+z^{2} g^{\prime \prime}(z)}}{z h^{\prime}(z)-\overline{z g^{\prime}(z)}}\right\} \geq \alpha, \quad z \in U .
$$

The classes of all harmonic starlike functions of order $\alpha$ and harmonic convex functions of order $\alpha$ are denoted by $S_{H}^{*}(\alpha)$ and $K_{H}(\alpha)$, respectively. These classes have been extensively studied by Jahangiri [8].

For $\alpha=0$, these classes $S_{H}^{*}(\alpha)$ and $K_{H}(\alpha)$ were denoted by $S_{H}^{*}$ and $K_{H}$, respectively and studied in detail by Silverman [14] and Silverman and Silvia [15], (see also [2]).

Now for $1<\beta \leq \frac{4}{3}$ and $z \in U$, suppose that $M_{H}(\beta)$ denote the family of harmonic functions $f=h+\bar{g}$ of the form (1.1) satisfying the condition

$$
\operatorname{Re}\left\{\frac{z h^{\prime}(z)-\overline{z g^{\prime}(z)}}{h(z)+\overline{g(z)}}\right\}<\beta,
$$

and $L_{H}(\beta)$ denote the family of harmonic functions of the form (1.1) satisfying the condition

$$
\operatorname{Re}\left\{1+\frac{z^{2} h^{\prime \prime}(z)+\overline{2 z g^{\prime}(z)+z^{2} g^{\prime \prime}(z)}}{z h^{\prime}(z)-\overline{z g^{\prime}(z)}}\right\}<\beta .
$$

Further, we let $V_{H}$ and $U_{H}$ be the subclasses of $S_{H}$ consisting of functions of the form

$$
f(z)=z+\sum_{k=2}^{\infty}\left|a_{k}\right| z^{k}-\sum_{k=1}^{\infty}\left|b_{k}\right| \bar{z}^{k}
$$

and

$$
f(z)=z+\sum_{k=2}^{\infty}\left|a_{k}\right| z^{k}+\sum_{k=1}^{\infty}\left|b_{k}\right| \bar{z}^{k}
$$

respectively.

Let $V_{H}^{*}(\alpha) \equiv S_{H}^{*}(\alpha) \cap V_{H}, V_{k}(\alpha) \equiv K_{H}(\alpha) \cap U_{H}, V_{H}(\beta) \equiv M_{H}(\beta) \cap V_{H}$ and $U_{H}(\beta) \equiv L_{H}(\beta) \cap U_{H}$. Further $V_{H}^{*}(0) \equiv V_{H}^{*}$ and $V_{K}(0) \equiv V_{K}$ are respectively the classes of starlike and convex functions in $V_{H}$ and $U_{H}$, respectively. 
We note that for $g \equiv 0$ the classes $M_{H}(\beta) \equiv M(\beta), L_{H}(\beta) \equiv L(\beta), V_{H}(\beta) \equiv$ $V(\beta)$ and $U_{H}(\beta) \equiv U(\beta)$ were studied by Uralegaddi et al. [16].

In 1975 Silverman [13] introduced a new class $T$ of analytic functions of the form $f(z)=z-\sum_{k=2}^{\infty}\left|a_{k}\right| z^{k}$ and opened up a new direction of studies in the theory of univalent functions. Uralegaddi et al. ([16], [17]) introduced analogous subclasses of starlike, convex and close-to-convex analytic functions with positive coefficients and opened up a new and interesting direction of research. In fact, they considered the functions where the coefficients are positive rather than negative real numbers. Motivated by the initial work of Uralegaddi et al. ([16], [17]) many researchers e.g. Dixit and Chandra [4], Dixit and Pathak [5], Porwal and Dixit[11] and Porwal et al. [12] introduced and studied various new subclasses of analytic functions with positive coefficients but analogues results on harmonic univalent functions have not been explored in the literature. Very recently Dixit and Porwal [6] attempt to fill this gap by introducing a new subclass of harmonic univalent functions with positive coefficients. In the present paper an attempt has been made to study in this direction. In this paper coefficient inequalities, extreme points, distortion bounds, covering theorems, convolution and convex combinations are determined for the classes $V_{H}(\beta)$ and $U_{H}(\beta)$. Further various inclusion relations are also obtained for these classes and discuss a class preserving integral operator.

\section{Main Results}

First, we give a sufficient coefficient bound for the class $M_{H}(\beta)$.

Theorem 2.1. Let the function $f=h+\bar{g}$ be given by (1.1). If

$$
\sum_{k=2}^{\infty} \frac{k-\beta}{\beta-1}\left|a_{k}\right|+\sum_{k=1}^{\infty} \frac{k+\beta}{\beta-1}\left|b_{k}\right| \leq 1,
$$

where $1<\beta \leq \frac{4}{3}$, then $f \in M_{H}(\beta)$.

Proof. Let

$$
\sum_{k=2}^{\infty} \frac{k-\beta}{\beta-1}\left|a_{k}\right|+\sum_{k=1}^{\infty} \frac{k+\beta}{\beta-1}\left|b_{k}\right| \leq 1 .
$$

It suffices to show that

$$
\left|\frac{\frac{z h^{\prime}(z)-\overline{z g^{\prime}(z)}}{h(z)+\overline{g(z)}}-1}{\frac{z h^{\prime}(z)-\overline{z g^{\prime}(z)}}{h(z)+\overline{g(z)}}-(2 \beta-1)}\right|<1, \quad z \in U .
$$


We have

$$
\begin{aligned}
& \quad\left|\frac{\frac{z h^{\prime}(z)-\overline{z g^{\prime}(z)}}{h(z)+\overline{g(z)}}-1}{\frac{z h^{\prime}(z)-\overline{z g^{\prime}(z)}}{h(z)+\overline{g(z)}}-(2 \beta-1)}\right| \\
& \leq \frac{\sum_{k=2}^{\infty}(k-1)\left|a_{k}\right||z|^{k-1}+\sum_{k=2}^{\infty}(k+1)\left|b_{k}\right||z|^{k-1}}{2(\beta-1)-\sum_{k=2}^{\infty}(k-2 \beta+1)\left|a_{k}\right||z|^{k-1}-\sum_{k=2}^{\infty}(k+2 \beta-1)\left|b_{k}\right||z|^{k-1}} \\
& \leq \frac{\sum_{k=2}^{\infty}(k-1)\left|a_{k}\right|+\sum_{k=2}^{\infty}(k+1)\left|b_{k}\right|}{2(\beta-1)-\sum_{k=2}^{\infty}(k-2 \beta+1)\left|a_{k}\right|-\sum_{k=2}^{\infty}(k+2 \beta-1)\left|b_{k}\right|} .
\end{aligned}
$$

The last expression is bounded above by 1 , if

$$
\sum_{k=2}^{\infty}(k-1)\left|a_{k}\right|+\sum_{k=2}^{\infty}(k+1)\left|b_{k}\right| \leq 2(\beta-1)-\sum_{k=2}^{\infty}(k-2 \beta+1)\left|a_{k}\right|-\sum_{k=2}^{\infty}(k+2 \beta-1)\left|b_{k}\right|,
$$

which is equivalent to

$$
\sum_{k=2}^{\infty} \frac{k-\beta}{\beta-1}\left|a_{k}\right|+\sum_{k=1}^{\infty} \frac{k+\beta}{\beta-1}\left|b_{k}\right| \leq 1 .
$$

But (2.2) is true by hypothesis.

Hence $\left|\frac{\frac{z h^{\prime}(z)-\overline{z g^{\prime}(z)}}{h(z)+\overline{g(z)}}-1}{\frac{z h^{\prime}(z)-\overline{z g^{\prime}(z)}}{h(z)+\bar{g}(z)}-(2 \beta-1)}\right|<1, z \in U$, and the theorem is proved.

Theorem 2.2. Let the function $f=h+\bar{g} \in S_{H}$ be given by (1.1). If

$$
\sum_{k=2}^{\infty} \frac{k(k-\beta)}{\beta-1}\left|a_{k}\right|+\sum_{k=1}^{\infty} \frac{k(k+\beta)}{\beta-1}\left|b_{k}\right| \leq 1,
$$

where $1<\beta \leq \frac{3}{2}$, then $f \in L_{H}(\beta)$.

Proof. The proof is similar to that of Theorem 2.1. Therefore we omit the details involved. $\square$ In the following theorem, it is proved that the condition (2.1) is also necessary for functions $f=h+\bar{g} \in V_{H}$ be given by (1.4).

Theorem 2.3. A function $f$ of the form (1.4) is in $V_{H}(\beta)$, if and only if

$$
\sum_{k=2}^{\infty}(k-\beta)\left|a_{k}\right|+\sum_{k=1}^{\infty}(k+\beta)\left|b_{k}\right| \leq \beta-1,
$$

where $1<\beta \leq \frac{4}{3}$.

Proof. Since $V_{H}(\beta) \subset M_{H}(\beta)$, we only need to prove the "only if" part of the theorem. For this we show that $f \notin V_{H}(\beta)$ if the condition (2.4) does not hold. 
Note that a necessary and sufficient condition for $f=h+\bar{g}$ given by (1.4) is in $V_{H}(\beta)$ if

$$
\operatorname{Re}\left\{\frac{z h^{\prime}(z)-\overline{z g^{\prime}(z)}}{h(z)+\overline{g(z)}}\right\}<\beta
$$

is equivalent to

$$
\operatorname{Re}\left\{\frac{(\beta-1) z-\sum_{k=2}^{\infty}(k-\beta)\left|a_{k}\right| z^{k}-\sum_{k=1}^{\infty}(k+\beta)\left|b_{k}\right| \bar{z}^{k}}{z+\sum_{k=2}^{\infty}\left|a_{k}\right| z^{k}-\sum_{k=1}^{\infty}\left|b_{k}\right| \bar{z}^{k}}\right\} \geq 0 .
$$

The above condition must hold for all values of $z,|z|=r<1$, Upon choosing the values of $z$ on the positive real axis where $0 \leq z=r<1$, we must have

$$
\frac{(\beta-1) z-\sum_{k=2}^{\infty}(k-\beta)\left|a_{k}\right| r^{k-1}-\sum_{k=1}^{\infty}(k+\beta)\left|b_{k}\right| r^{k-1}}{1+\sum_{k=2}^{\infty}\left|a_{k}\right| r^{k-1}-\sum_{k=1}^{\infty}\left|b_{k}\right| r^{k-1}} \geq 0
$$

If the condition (2.4) does not hold then the numerator of (2.5) is negative for $r$ sufficiently close to 1 . Thus there exists a $z_{0}=r_{0}$ in $(0,1)$ for which the quotient in (2.5) is negative. This contradicts the required condition for $f \in V_{H}(\beta)$ and so the proof is complete.

Theorem 2.4. A function $f$ of the form (1.5) is in $U_{H}(\beta)$. If and only if

$$
\sum_{k=2}^{\infty} k(k-\beta)\left|a_{k}\right|+\sum_{k=1}^{\infty} k(k+\beta)\left|b_{k}\right| \leq \beta-1,
$$

where $1<\beta \leq \frac{3}{2}$.

Proof. The proof is much akin to that of Theorem 2.3. Therefore we omit the details involved.

Next, we determine the extreme points of the closed convex hulls of $V_{H}(\beta)$, denoted by $\operatorname{clco} V_{H}(\beta)$.

Theorem 2.5. $f \in$ clco $V_{H}(\beta)$, if and only if

$$
f(z)=\sum_{k=1}^{\infty}\left\{x_{k} h_{k}(z)+y_{k} g_{k}(z)\right\}
$$

where $h_{1}(z)=z, h_{k}(z)=z+\frac{\beta-1}{k-\beta} z^{k},(k=2,3, \ldots)$ and $g_{k}(z)=z+\frac{\beta-1}{k+\beta} z^{k}$, $(k=1,2, \ldots), \sum_{k=1}^{\infty}\left(x_{k}+y_{k}\right)=1, x_{k} \geq 0, y_{k} \geq 0$. In particular, the extreme points of $V_{H}(\beta)$ are $\left\{h_{k}\right\}$ and $\left\{g_{k}\right\}$.

Proof. For functions $f$ of the form (2.7), we have

$$
\begin{aligned}
f(z) & =\sum_{k=1}^{\infty}\left\{x_{k} h_{k}(z)+y_{k} g_{k}(z)\right\} \\
& =z+\sum_{k=2}^{\infty} \frac{\beta-1}{k-\beta} x_{k} z^{k}-\sum_{k=1}^{\infty} \frac{\beta-1}{k+\beta} y_{k} \bar{z}^{k} .
\end{aligned}
$$


Then

$$
\begin{aligned}
& \sum_{k=2}^{\infty} \frac{k-\beta}{\beta-1}\left\{\frac{\beta-1}{k-\beta} x_{k}\right\}+\sum_{k=1}^{\infty} \frac{k+\beta}{\beta-1}\left\{\frac{\beta-1}{k+\beta} y_{k}\right\} \\
= & \sum_{k=2}^{\infty} x_{k}+\sum_{k=1}^{\infty} y_{k} \\
= & 1-x_{1} \leq 1,
\end{aligned}
$$

and so $f \in \operatorname{clco} V_{H}(\beta)$. Set $x_{k}=\frac{k-\beta}{\beta-1}\left|a_{k}\right|, k=2,3,4, \ldots$ and $y_{k}=\frac{k+\beta}{\beta-1}\left|b_{k}\right|, k=$ $1,2,3, \ldots$

Then note that by Theorem 2.3, $0 \leq x_{k} \leq 1,(k=2,3,4, \ldots)$ and $0 \leq y_{k} \leq$ $1,(k=1,2,3, \ldots)$. We define $x_{1}=1-\sum_{k=2}^{\infty} x_{k}-\sum_{k=1}^{\infty} y_{k}$, and by Theorem 2.3 , $x_{1} \geq 0$.

Consequently, we obtain $f(z)=\sum_{k=1}^{\infty}\left\{x_{k} h_{k}(z)+y_{k} g_{k}(z)\right\}$ as required.

Theorem 2.6. $f \in$ clco $U_{H}(\beta)$, if and only if

$$
f(z)=\sum_{k=1}^{\infty}\left\{x_{k} h_{k}(z)+y_{k} g_{k}(z)\right\}
$$

where $h_{1}(z)=z, h_{k}(z)=z+\frac{\beta-1}{k(k-\beta)} z^{k},(k=2,3, \ldots)$ and $g_{k}(z)=z+\frac{\beta-1}{k(k+\beta)} z^{k}$, $(k=1,2, \ldots), \sum_{k=1}^{\infty}\left(x_{k}+y_{k}\right)=1, x_{k} \geq 0, y_{k} \geq 0$. In particular, the extreme points of $U_{H}(\beta)$ are $\left\{h_{k}\right\}$ and $\left\{g_{k}\right\}$.

Proof. The proof is much akin to that of Theorem 2.5. Therefore we omit the details involved.

Theorem 2.7. Let $f \in V_{H}(\beta)$. Then for $|z|=r<1$, we have

$$
|f(z)| \leq\left(1+\left|b_{1}\right|\right) r+\left(\frac{\beta-1}{2-\beta}-\frac{\beta+1}{2-\beta}\left|b_{1}\right|\right) r^{2}
$$

and

$$
|f(z)| \geq\left(1-\left|b_{1}\right|\right) r-\left(\frac{\beta-1}{2-\beta}-\frac{\beta+1}{2-\beta}\left|b_{1}\right|\right) r^{2} .
$$

Proof. We only prove the right hand inequality. The proof for left hand inequality is similar and will be omitted. Let $f(z) \in V_{H}(\beta)$. Taking the absolute value of $f$, 
we have

$$
\begin{aligned}
|f(z)| & \leq\left(1+\left|b_{1}\right|\right) r+\sum_{k=2}^{\infty}\left(\left|a_{k}\right|+\left|b_{k}\right|\right) r^{k} \\
& \leq\left(1+\left|b_{1}\right|\right) r+\sum_{k=2}^{\infty}\left(\left|a_{k}\right|+\left|b_{k}\right|\right) r^{2} \\
& =\left(1+\left|b_{1}\right|\right) r+\frac{\beta-1}{2-\beta} \sum_{k=2}^{\infty}\left(\frac{2-\beta}{\beta-1}\left|a_{k}\right|+\frac{2-\beta}{\beta-1}\left|b_{k}\right|\right) r^{2} \\
& \leq\left(1+\left|b_{1}\right|\right) r+\frac{\beta-1}{2-\beta} \sum_{k=2}^{\infty}\left(\frac{k-\beta}{\beta-1}\left|a_{k}\right|+\frac{k+\beta}{\beta-1}\left|b_{k}\right|\right) r^{2} \\
& \leq\left(1+\left|b_{1}\right|\right) r+\frac{\beta-1}{2-\beta}\left(1-\frac{\beta+1}{\beta-1}\left|b_{1}\right|\right) r^{2} \\
& =\left(1+\left|b_{1}\right|\right) r+\left(\frac{\beta-1}{2-\beta}-\frac{\beta+1}{2-\beta}\left|b_{1}\right|\right) r^{2} .
\end{aligned}
$$

Theorem 2.8. Let $f \in U_{H}(\beta)$. Then for $|z|=r<1$, we have

$$
|f(z)| \leq\left(1+\left|b_{1}\right|\right) r+\left(\frac{\beta-1}{2-\beta}-\frac{\beta+1}{2-\beta}\left|b_{1}\right|\right) \frac{r^{2}}{2}
$$

and

$$
|f(z)| \geq\left(1-\left|b_{1}\right|\right) r-\left(\frac{\beta-1}{2-\beta}-\frac{\beta+1}{2-\beta}\left|b_{1}\right|\right) \frac{r^{2}}{2} .
$$

Proof. The proof follows as that of Theorem 2.7.

The following covering results follow from the left hand inequality in Theorem 2.7 and Theorem 2.8 for the classes $V_{H}(\beta)$ and $U_{H}(\beta)$, respectively.

Corollary 2.9. Let $f$ of the form (1.4) be so that $f \in V_{H}(\beta)$. Then

$$
\left\{\omega:|\omega|<\frac{3-2 \beta}{2-\beta}+\frac{2 \beta-1}{2-\beta}\left|b_{1}\right|\right\} \subset f(U) \text {. }
$$

Corollary 2.10. Let of the form (1.5) be so that $f \in U_{H}(\beta)$. Then

$$
\left\{\omega:|\omega|<\frac{5-2 \beta}{2(2-\beta)}+\frac{3(\beta-1)}{2(2-\beta)}\left|b_{1}\right|\right\} \subset f(U) .
$$

For our next theorem, we need to define the convolution of two harmonic functions. For harmonic functions of the form

$$
f(z)=z+\sum_{k=2}^{\infty}\left|a_{k}\right| z^{k}-\sum_{k=1}^{\infty}\left|b_{k}\right| \bar{z}^{k}
$$


and

$$
F(z)=z+\sum_{k=2}^{\infty}\left|A_{k}\right| z^{k}-\sum_{k=1}^{\infty}\left|B_{k}\right| \bar{z}^{k}
$$

we define the convolution of two harmonic functions $f$ and $F$ as

$$
(f * F)(z)=f(z) * F(z)=z+\sum_{k=2}^{\infty}\left|a_{k} A_{k}\right| z^{k}-\sum_{k=1}^{\infty}\left|b_{k} B_{k}\right| \bar{z}^{k} .
$$

Using this definition, we show that the class $V_{H}(\beta)$ is closed under convolution.

Theorem 2.11. For $1<\alpha \leq \beta \leq \frac{4}{3}$, let $f \in V_{H}(\alpha)$ and $F \in V_{H}(\beta)$. Then $f * F \in V_{H}(\alpha) \subseteq V_{H}(\beta)$.

Proof. Let $f(z)=z+\sum_{k=2}^{\infty}\left|a_{k}\right| z^{k}-\sum_{k=1}^{\infty}\left|b_{k}\right| \bar{z}^{k}$ be in $V_{H}(\alpha)$ and $F(z)=z+$ $\sum_{k=2}^{\infty}\left|A_{k}\right| z^{k}-\sum_{k=1}^{\infty}\left|B_{k}\right| \bar{z}^{k}$ be in $V_{H}(\beta)$.

Then the convolution $f * F$ is given by (2.9). We wish to show that the coefficients of $f * F$ satisfy the required condition given in Theorem 2.3. For $F(z) \in V_{H}(\beta)$ we note that $\left|A_{k}\right| \leq 1$ and $\left|B_{k}\right| \leq 1$. Now, for the convolution function $f * F$, we obtain

$$
\begin{aligned}
& \sum_{k=2}^{\infty} \frac{k-\beta}{\beta-1}\left|a_{k} A_{k}\right|+\sum_{k=1}^{\infty} \frac{k+\beta}{\beta-1}\left|b_{k} B_{k}\right| \\
\leq & \sum_{k=2}^{\infty} \frac{k-\beta}{\beta-1}\left|a_{k}\right|+\sum_{k=1}^{\infty} \frac{k+\beta}{\beta-1}\left|b_{k}\right| \\
\leq & 1, \quad \text { since } f(z) \in V_{H}(\beta) .
\end{aligned}
$$

Therefore $f * F \in V_{H}(\alpha) \subseteq V_{H}(\beta)$.

Theorem 2.12. For $1<\alpha \leq \beta \leq \frac{3}{2}$ let $f \in U_{H}(\alpha)$ and $F \in U_{H}(\beta)$. Then $f * F \in U_{H}(\alpha) \subseteq U_{H}(\beta)$.

Proof. The proof follows as that of Theorem 2.11.

Theorem 2.13. The class $V_{H}(\beta)$ is closed under convex combination.

Proof. For $i=1,2,3, \ldots$ let $f_{i}(z) \in V_{H}(\beta)$, where $f_{i}(z)$ is given by

$$
f_{i}(z)=z+\sum_{k=2}^{\infty}\left|a_{k_{i}}\right| z^{k}-\sum_{k=1}^{\infty}\left|b_{k_{i}}\right| \bar{z}^{k}
$$

Then by Theorem 2.3,

$$
\sum_{k=2}^{\infty} \frac{k-\beta}{\beta-1}\left|a_{k_{i}}\right|+\sum_{k=1}^{\infty} \frac{k+\beta}{\beta-1}\left|b_{k_{i}}\right| \leq 1 .
$$


For $\sum_{i=1}^{\infty} t_{i}=1,0 \leq t_{i} \leq 1$, the convex combination of $f_{i}$ may be written as

$$
\sum_{i=1}^{\infty} t_{i} f_{i}(z)=z+\sum_{k=2}^{\infty}\left(\sum_{i=1}^{\infty} t_{i}\left|a_{k_{i}}\right|\right) z^{k}-\sum_{k=1}^{\infty}\left(\sum_{i=1}^{\infty} t_{i}\left|b_{k_{i}}\right|\right) \bar{z}^{k} .
$$

Then by (2.10), we have

$$
\begin{aligned}
& \sum_{k=2}^{\infty} \frac{k-\beta}{\beta-1}\left(\sum_{i=1}^{\infty} t_{i}\left|a_{k_{i}}\right|\right)+\sum_{k=1}^{\infty} \frac{k+\beta}{\beta-1}\left(\sum_{i=1}^{\infty} t_{i}\left|b_{k_{i}}\right|\right) \\
= & \sum_{i=1}^{\infty} t_{i}\left(\sum_{k=2}^{\infty} \frac{k-\beta}{\beta-1}\left|a_{k_{i}}\right|+\sum_{k=1}^{\infty} \frac{k+\beta}{\beta-1}\left|b_{k_{i}}\right|\right) \\
\leq & \sum_{i=1}^{\infty} t_{i}=1 .
\end{aligned}
$$

This is the condition required by Theorem 2.3 and so $\sum_{i=1}^{\infty} t_{i} f_{i}(z) \in V_{H}(\beta)$.

The proof of following theorem is similar to Theorem 2.13, so we state it only.

Theorem 2.14. The class $U_{H}(\beta)$ is closed under convex combination.

\section{Inclusion Relations}

To prove our next theorem, we shall require the following lemma due to Jahangiri [8].

Lemma 3.1. Let $f=h+\bar{g} \in V_{H}$ be given by (1.4) and if

$$
\sum_{k=2}^{\infty} \frac{k-\alpha}{1-\alpha}\left|a_{k}\right|+\sum_{k=1}^{\infty} \frac{k+\alpha}{1-\alpha}\left|b_{k}\right| \leq 1, \quad(0 \leq \alpha<1) .
$$

Then $f \in V_{H}^{*}(\alpha)$.

Theorem 3.1. If $f \in V_{H}(\beta)$ then $f \in V_{H}^{*}\left(\frac{4-3 \beta}{3-2 \beta}\right)$.

Proof. Since $f \in V_{H}(\beta)$ then by Theorem 2.3 we must have

$$
\sum_{k=2}^{\infty} \frac{k-\beta}{\beta-1}\left|a_{k}\right|+\sum_{k=1}^{\infty} \frac{k+\beta}{\beta-1}\left|b_{k}\right| \leq 1 .
$$

To show that $f \in V_{H}^{*}\left(\frac{4-3 \beta}{3-2 \beta}\right)$, by virtue of Lemma 3.1 we have to show that

$$
\sum_{k=2}^{\infty} \frac{k-\frac{4-3 \beta}{3-2 \beta}}{1-\frac{4-3 \beta}{3-2 \beta}}\left|a_{k}\right|+\sum_{k=1}^{\infty} \frac{k+\frac{4-3 \beta}{3-2 \beta}}{1-\frac{4-3 \beta}{3-2 \beta}}\left|b_{k}\right| \leq 1, \quad\left(0 \leq \frac{4-3 \beta}{3-2 \beta}<1\right) .
$$


For this it is sufficient to prove that

$$
\frac{k-\beta}{\beta-1} \geq \frac{k-\frac{4-3 \beta}{3-2 \beta}}{1-\frac{4-3 \beta}{3-2 \beta}}, \quad(k=2,3,4, \ldots)
$$

and

$$
\frac{k+\beta}{\beta-1} \geq \frac{k+\frac{4-3 \beta}{3-2 \beta}}{1-\frac{4-3 \beta}{3-2 \beta}}, \quad(k=1,2,3, \ldots)
$$

or equivalently

$$
(\beta-1)(k-\beta) \geq 0,(k=2,3,4, \ldots) \text { and }(\beta-1)(k+\beta) \geq 0,(k=1,2,3, \ldots),
$$

which is true and the theorem is proved.

The proof of following theorem is similar to Theorem 3.1, so we state it only.

Theorem 3.2. If $f \in U_{H}(\beta)$ then $f \in V_{k}\left(\frac{4-3 \beta}{3-2 \beta}\right)$.

Following corollaries are an easy consequences of Theorem 3.1 and 3.2.

Corollary 3.3. $V_{H}(\beta) \subset V_{H}\left(\frac{4}{3}\right) \subset V_{H}^{*}$.

Corollary 3.4. $U_{H}(\beta) \subset U_{H}\left(\frac{3}{2}\right) \subset V_{k}$.

Corollary 3.5. $U_{H}\left(\frac{4}{3}\right) \subset V_{H}\left(\frac{6}{5}\right)$.

Corollary 3.6. $U_{H}\left(\frac{4}{3}\right) \subset V_{H}^{*}\left(\frac{2}{3}\right)$.

\section{A Family of Class Preserving Integral Operator}

Let $f(z)=h(z)+\overline{g(z)}$ be defined by (1.1). Let us define $F(z)$ by the relation

$$
F(z)=\frac{c+1}{z^{c}} \int_{0}^{z} t^{c-1} h(t) d t+\overline{\frac{c+1}{z^{c}} \int_{0}^{z} t^{c-1} g(t) d t}, \quad(c>-1) .
$$

Theorem 4.1. Let $f(z)=h(z)+\overline{g(z)} \in S_{H}$ be given by (1.4) and $f \in V_{H}(\beta)$, where $1<\beta \leq \frac{4}{3}$. Then $F(z)$ defined by (4.1) is also in the class $V_{H}(\beta)$.

Proof. Let $f(z)=z+\sum_{k=2}^{\infty}\left|a_{k}\right| z^{k}-\sum_{k=1}^{\infty}\left|b_{k}\right| \bar{z}^{k}$ be in $V_{H}(\beta)$ then by Theorem 2.3 , we have

$$
\sum_{k=2}^{\infty} \frac{k-\beta}{\beta-1}\left|a_{k}\right|+\sum_{k=1}^{\infty} \frac{k+\beta}{\beta-1}\left|b_{k}\right| \leq 1 .
$$

From the representation (4.1) of $F(z)$, it follows that

$$
F(z)=z+\sum_{k=2}^{\infty} \frac{c+1}{c+k}\left|a_{k}\right| z^{k}-\sum_{k=1}^{\infty} \frac{c+1}{c+k}\left|b_{k}\right| \bar{z}^{k}
$$


Now

$$
\begin{aligned}
& \sum_{k=2}^{\infty} \frac{k-\beta}{\beta-1}\left(\frac{c+1}{c+k}\right)\left|a_{k}\right|+\sum_{k=1}^{\infty} \frac{k+\beta}{\beta-1}\left(\frac{c+1}{c+k}\right)\left|b_{k}\right| \\
\leq & \sum_{k=2}^{\infty} \frac{k-\beta}{\beta-1}\left|a_{k}\right|+\sum_{k=1}^{\infty} \frac{k+\beta}{\beta-1}\left|b_{k}\right| \\
\leq & 1 .
\end{aligned}
$$

Thus $F(z) \in V_{H}(\beta)$.

The proof of following theorem is similar to Theorem 4.1, so we state it only.

Theorem 4.2. Let $f(z)=h(z)+\overline{g(z)} \in S_{H}$ be given by (1.5) and $f \in U_{H}(\beta)$, where $1<\beta \leq \frac{3}{2}$. Then $F(z)$ defined by (4.1) is also in the class $U_{H}(\beta)$.

Acknowledgements. The authors are thankful to the referee for his valuable comments and suggestions.

\section{References}

[1] O. P. Ahuja, Planar harmonic univalent and related mappings, J. Inequal. Pure Appl. Math., 6(4)(2005), Art. 122, 1-18.

[2] Y. Avci and E. Zlotkiewicz, On harmonic univalent mappings, Ann. Univ. Mariae Curie-Sklodowska Sect., A44(1990), 1-7.

[3] J. Clunie and T. Sheil-Small, Harmonic univalent functions, Ann. Acad. Sci. Fenn. Ser.AI Math., 9(1984), 3-25.

[4] K. K. Dixit and Vikas Chandra, On subclass of univalent functions with positive coefficients, Aligarh Bull. Math., 27(2)(2008), 87-93.

[5] K. K. Dixit and A. L. Pathak, A new class of analytic functions with positive coefficients, Indian J. Pure Appl. Math., 34(2)(2003), 209-218.

[6] K. K. Dixit and Saurabh Porwal, A subclass of harmonic univalent functions with positive coefficients, Tamkang J. Math., 41(3)(2010), 261-269.

[7] P. Duren, Harmonic mappings in the plane, Camb. Univ. Press, (2004).

[8] J. M. Jahangiri, Harmonic functions starlike in the unit disc, J. Math. Anal. Appl., 235(1999), 470-477.

[9] S. Ponnusamy and A. Rasila, Planar harmonic mappings, Ramanujan Mathematical Society Mathematics Newsletters, 17(2)(2007), 40-57.

[10] S. Ponnusamy and A. Rasila, Planar harmonic and quasi-conformal mappings, Ramanujan Mathematical Society Mathematics Newsletters, 17(3)(2007), 85-101.

[11] Saurabh Porwal and K. K. Dixit, An application of certain convolution operator involving hypergeometric functions, J. Raj. Acad. Phy. Sci., 9(2)(2010), 173-186. 
[12] Saurabh Porwal, K. K. Dixit, Vinod Kumar and Poonam Dixit, On a subclass of analytic functions defined by convolution, General Mathematics, General Mathematics, 19(3)(2011), 57-65.

[13] H. Silverman, Univalent functions with negative coefficients, Proc. Amer. Math. Soc., 51(1975), 109-116.

[14] H. Silverman, Harmonic univalent function with negative coefficients, J. Math. Anal. Appl., 220(1998), 283-289.

[15] H. Silverman, E. M. Silvia, Subclasses of Harmonic univalent functions, New Zealand. J. Math., 28(1999), 275-284.

[16] B. A. Uralegaddi, M. D. Ganigi and S. M. Sarangi, Univalent functions with positive coefficients, Tamkang J. Math., 25(1994), 225-230.

[17] B. A. Uralegaddi, M. D. Ganigi and S. M. Sarangi, Close-to-Convex functions with positive coefficients, Studia Univ. Babes-Balyai, Mathematica, XL4(1995), 25-31. 